\title{
Phenotypic Detection of Extended Spectrum Beta lactamase and Carbapenemase Co-producing Clinical Isolates from Two Tertiary Hospitals in Kano, North West Nigeria
}

\author{
Yusuf Ibrahim ${ }^{1 *}$, Yahaya Sani ${ }^{1}$, Qabli Saleh $^{2}$, Algarni Saleh ${ }^{2}$, Gbadamosi \\ Hakeem $^{3}$
}

OPEN ACCESS

Citation: Yusuf Ibrahim, Yahaya Sani, Qabli Saleh, Algarni Saleh, Gbadamosi Hakeeml. Phenotypic Detection of Extended Spectrum Beta lactamase and Carbapenemase Co-producing Clinical Isolates from Two Tertiary Hospitals in Kano, North West Nigeria. Ethiop J Health Sci 2017;27(1):3-10. doi: http://dx.doi.org/10.4314/ejhs.v27i1.2 Received: September 15, 2015

Accepted: December 1, 2015

Published: January 1, 2017

Copyright: Yusuf I, et al. This is an open access article distributed under the terms of the Creative Commons Attribution License, which permits unrestricted use, distribution, and reproduction in any medium, provided the original author and source are credited.

Funding: Nil

Competing Interests: The authors

declare that this manuscript was approved by all authors in its form and that no competing interest exists.

Affiliation and Correspondence:

${ }^{1}$ Department of Microbiology, Faculty of Science, Bayero University, Kano, Nigeria

${ }^{2}$ Department of Microbiology, King Abdulaziz University, Jeddah, Saudi Arabia

${ }^{3}$ Federal Medical Center, Katsina, Nigeria

"Email: iyusuf.bio@buk.edu.ng

\section{ABSTRACT}

BACKGROUND: Continue rise in unprofessional use of antibiotics in our hospitals and communities is worrisome. A research study was therefore conducted to detect extended spectrum beta-lactamase (ESBL), carbapenemase, metallobeta lactamase and their co-production phenotypically from isolates obtained from patients admitted to or attending two tertiary hospitals in Kano, Nigeria.

METHOD: A total of 248 isolates of Escherichia coli and Klebsiella pneumoniaewere screened phenotypically for ESBL production and carbapenemase production according to CLS1 2012 breakpoints using double disk synergy test and modified Hodge test (MHT) respectively. Antibiotic susceptibility of the organisms was tested against colistin, tigecycline and 3 flouroquinolones.

RESULT: The result shows that 58.0\% of the isolates were ESBL producers with higher percentage in $K$. pneumoniae (62.9\%). Further, about $40.3 \%$ and $36.6 \%$ of the isolates were resistant to meropenem and imipenem respectively. However, E. coli showed higher resistance to meropenem (47.1\%) while $K$. pneumoniae showed higher resistance to imipenem (44.4\%). Co-productions of carbapenemase and ESBL were observed in both $E$. coli and $K$. pneumoniae. Carbapenemase producing isolates were more obtained from uro-pathogens and wound isolates, with almost all the cases of co-production of the $\beta$ lactamases occurring in urine and cathertips isolates. Overall susceptibilities of the isolates to colistin and tigecycline were 64.6 and $70.0 \%$ respectively, but isolates were less susceptible to flouroquinolones.

CONCLUSION: The finding of the study therefore indicates that carbapenem resistance is mediated by carbapenemase production and or overproduction of ESBL coupled with reduced porins. Coproduction of carbapenemase, MBLs and ESBLs by some of the isolates is worrisome. Susceptibility to colistin and tigecycline was still promising, but increasing resistance to flouroquinolones has been observed.

KEYWORDS: Carbapenemase, ESBL, colistin, tigecycline, flouroquinolones, phenotypic, Kano 


\section{INTRODUCTION}

Hospital wards, especially those in low income countries where infection prevention and control (IPC) protocols are lacking have become the main foci for developing multidrug resistance such as multidrug-resistant Gram negative bacilli (1). Community acquisitions of these hard to treat bacteria are highly possible due to inadequate environmental/personal sanitations and lack of surveillance (1).

Increasing rates of Gram negative bacteria resistance to beta lactam antibiotics such as cephalosporins and carbapenems are of great concern not only to health care workers (HCWs) but to the public. This is because they are the drug of choice for the treatment of very stubborn infection by many Gram negative bacteria (2). However, the increasing use of these antibiotics in some countries has led to the development of carbapenem resistant pathogens, which have already found their ways to low income countries where treatments with such antibiotics (carbapenems) are not frequent. However, carbapenem-resistant strains are emerging worldwide, and resistance rates have reached 50$60 \%$ in some institutions (3). However, data regarding carbapenemase and other beta lactamase mediated resistance are scarce in overburden low income healthcare facilities. This paucity of information in our facilities poses a great danger to general health care delivery system (4-6).

During the last decade, carbapenem resistance has been increasingly reported among Enterobacteriaceae and is largely attributed to the production of class A $\beta$-lactamase, the Klebsiella pneumoniae carbapenemase (KPC) and Ambler class B metallo- $\beta$-lactamases (MBLs) requiring metal ion for action (7). In addition, some clinical isolates like E. coli and $K$. pneumoniae are capable of co-producing both MBL and KPC carbapenemases in addition to ESBL, which may further narrow down treatment options with $\beta$ lactam and non- $\beta$-lactam antibiotics.

The mechanism of carbapenem resistance is either through the acquisition of carbapenemhydrolysing $\beta$-lactamases like carbapenemase or through production of ESBL in association with impaired permeability via loss of porins
(8).Infections caused by organisms producing such enzymes have resulted in poor outcomes, reduced rate of clinical and microbiological responses, longer hospital stays and greater hospital expenses (9). Physical contact is the most likely mode of transmission and the gastrointestinal tract of colonized or infected patients is the most frequent reservoir while transient carriage of bacteria on the hands of healthcare workers may lead to transmission to patients (10).

The lack of screening for these multi-drug resistant pathogens in our hospitals coupled with poor antibiotic stewardship and surveillance systemshas increased their burden in our hospitals and community in recent years. The traditional susceptibility testing protocol being used currently in many clinical laboratories in Nigeria cannot detect bacteria harboring these enzymes. This will eventually lead to inappropriate diagnosis, unsuccessful therapy of patient and unnecessary use of drugs.Since molecular detection and genotyping of resistant isolates are expensive and unavailable in most hospitals, this study aimed to detect carbapenemase and ESBL production among the clinical isolates phenotypically in the two tertiary hospitals in Nigeria and to relate the gross antibiotic resistance being observed in the hospitals to carbapenemase and ESBL or coproduction of two or more by the pathogens, and to test the susceptibility of the carbapenem and ESBL producing pathogens to colistin and tigecycline.

\section{MATERIALS AND METHOD}

Collection of Clinical Isolates: A total of two hundred and forty-eight (248) consecutive nonrepetitive clinical bacterial isolates of Escherichia coli (140) and Klebsiella pneumoniae (108) were derived from samples of urine, catheter tips and wounds, and abscesses were collected from pathology department of the hospital A and B over a period of 12 months. Hospital A (Aminu Kano Teaching Hospital) is a teaching hospital with about 600bedcapacity while hospital B (Murtala Muhammad Specialist Hospital, Kano) is a general specialist hospital with about 700 bed capacity. The two hospitals receive patients from all over the country and neighboring countries. The demographic information and the history of

DOI: http://dx.doi.org/10.4314/ejhs.v27i1.2 
each patient was obtained from his/her records. The isolation and identification of the bacterial pathogens were made in the laboratory by the routine standard method used to identify pathogens in the two hospitals. All urine and cathertip samples were cultured on cysteine lactose electrolyte deficient (CLED) and nutrient agar (NA) while wound and abscess samples were cultured on blood, nutrient and McConkey agar for bacterial isolation. Gram negative bacterial pathogens isolated were identified by biochemical tests according to CLSI protocols. The following antibiotics were tested against the isolates in the laboratory as part of their routine practice using by Kirby-Bauer's disc diffusion method: gentamicin $(10 \mu \mathrm{g})$, co-trimoxazole $(25 \mu \mathrm{g})$, ciprofloxacin $(5$ $\mu \mathrm{g})$, cefpodoxime $(30 \mu \mathrm{g})$, ceftazidime $(30 \mu \mathrm{g})$, ceftriaxone $(30 \mu \mathrm{g})$, cefoxitin $(30 \mu \mathrm{g})$, nalidixic acid $(30 \mu \mathrm{g})$, amoxycillin $(20 \mu \mathrm{g})$, nitrofurantoin $(300 \mu \mathrm{g})$, tetracycline $(30 \mu \mathrm{g})$, chlorampenicol (30 $\mu \mathrm{g})$ and augmentin $(30 \mu \mathrm{g})$. Isolated pathogens included E. coli, K. pneumoniae, Serratia sp, Proteus sp, Citrobacter sp. and Pseudomonasaeruginosa. Only $K$. pneumoniae and $E$. coli were selected because they were the highest prevalent uropathogen and of wound and abscess in the two hospitals, as shown in previous studies, and they showed high resistance to many of the routine antibiotics in addition to $P$. aeruginosa. Because few $P$. aeruginosa was obtained, it was not included in the study. However, during the selection, attention was given to isolates that were at least resistant to 3 classes of antibiotics including beta-lactams.

\section{Test for Extended Spectrum Beta} lactamase (ESBL): All the clinical pathogens were screened for ESBLs using phenotypic methods according to CLSI guidelines. In the presumptive test to detect potential ESBL producers, all the isolates were screened for susceptibility to cefpodoxime $(10 \mu \mathrm{g})$,ceftazidime $(30 \mu \mathrm{g})$ and the ceftriaxone $(30 \mu \mathrm{g})$ antibiotic discs (Oxoid, UK). Results were interpreted based on the CLSI guidelines as follows: zones of inhibition of $\leq 17 \mathrm{~mm}$ for cefpodoxime; $\leq 22 \mathrm{~mm}$ for ceftazidime and $\leq 25 \mathrm{~mm}$ ceftriaxone or combination of two or more. The less susceptible or resistant isolates were subjected to confirmatory test using the double discs synergy test as described in the CLSI guidelines.

Carbapenem Susceptibility Testing: Susceptibility of all the isolates to carbapenems was tested in accordance with the current CLSI guidelines (11). Carbapenem antibiotics MEM and IPM were used. The discs were placed on the surface of inoculated Mueller Hinton Agar (MHA) (Hi media, India) plates using sterile forceps. The discs were placed about $25 \mathrm{~mm}$ apart, and the plates were incubated for 24 hours at $37{ }^{\circ} \mathrm{C}$ after which zones of inhibition in diameter were recorded using a ruler according to CDC guidelines (12). E. coli ATCC 25922 strain was used as a control culture. Isolates that showed a zone of inhibition $\leq 21 \mathrm{~mm}$ in diameter for meropenem or $\leq 23 \mathrm{~mm}$ in diameter for imipenem were considered as carbapenem resistant.

\section{Screening for carbapenemase production}

The selected carbapenem-resistant pathogens were screened for carbapenemase production phenotypically using Modified Hodge Test (MHT) as recommended by the CLSI guidelines (12). This is necessary in order to determine if the reduced susceptibility to carbapenems observed is mediated by a $\beta$ lactamase-carbapenemase.

Detection of class $B$ metallo B-Lactamases (MBLs): The carbapenemase producing organisms detected where further screened for Class B metallo-carbapenemases (MBLs), which are characterised by inhibition by metal chelators, EDTA. EDTA-disc synergy based method described by Walsh et al (13) and CLSI (11) was employed. The method involves streaking a carbapenemase producing isolate on MHA, followed by placing two MEM discs; one containing EDTA and the other without EDTA at a distance of about $30 \mathrm{~mm}$ apart. The EDTA disc was prepared in-house by adding $250 \mu$ l of neutral EDTA to MEM. The plates were incubated for 24 hours at $37{ }^{\circ} \mathrm{C}$ and read for zones of inhibition after incubation. Control disc containing EDTA alone was used to determine the activity of the EDTA to ensure that it does not cause falsepositive results by inhibiting the test isolate. Organisms that showed difference in diameter of zone of inhibition by $\geq 4 \mathrm{~mm}$ on the MEM containing EDTA and MEM alone were confirmed for the production of MBLs. 
Screening of Carbapenemase, MBL and ESBL Producers for Susceptibility to Colistin, Tigecycline and Flouroquinolones: The susceptibility of the carbapenemase (MBL inclusive) and ESBL producers to colistin $(10 \mu \mathrm{g})$, tigecycline $(15 \mu \mathrm{g})$, ciprofloxacin $(5 \mu \mathrm{g})$, levofloxacin $(30 \mu \mathrm{g})$ and ofloxacin $(30 \mu \mathrm{g})$ (Oxoid UK), was performed by disc diffusion method. The zone diameters of each of the antibiotics were interpreted as per CLSI 2012recommendations. Isolates producing a diameter of $\geq 14 \mathrm{~mm}$ and $\geq$ $21 \mathrm{~mm}$ for colistin and tigecycline respectively were considered susceptible. E. coli ATCC 25922 was used as the control strain for the antimicrobial susceptibility testing.

\section{RESULTS}

Distribution and carbapenem-susceptibility of the isolates: The distribution of the 248 isolates obtained from patients from the two hospitals were $140 \mathrm{E}$. coli $(56.5 \%)$ and $108 \mathrm{~K}$. pneumoniae (43.5\%), which were obtained from patients with a mean age of 37.3 years.Sixty-six (66) of the $E$. coli and 59 of the $K$. pneumoniae were obtained from surgical wards, 28 and 33 from out patients (OP) while the rest of $46 \mathrm{E}$. coli and $16 \mathrm{~K}$. pneumoniaewere obtained from other wards (inpatients). Most of the isolates were from urine and cathetertip samples $(83 \mathrm{E}$. coli and $61 \mathrm{~K}$. pneumoniae), and others included wound swabs (16E. coli and $25 K$. pneumoniae) and abscess (41 E. coli and $24 K$. pneumoniae.

Of the total 248 isolates screened for ESBL production, $58.0 \%$ were ESBL producers with higher percentage in $K$. pneumoniae $(62.9 \%)$. Meanwhile, a total percentage of $40.3 \%$ and $36.6 \%$ of the isolates were resistant to MEM and IMP respectively with $E$. coli showing higher resistance to MEM (47.1\%) and $K$. pneumoniae showing higher resistance to IMP (44.4\%) (Table 1). In out of the $66 \mathrm{E}$. coli that showed positive cut-off zone breakpoints for MEM, 32 (48.5\%) of the isolates were also resistant to IMP, but in the case of $K$. pneumoniae, $23(67.6 \%)$ were also resistant to IMP.

Table 1: resistance of the pathogens to carbapenems

\begin{tabular}{lcccc}
\hline $\begin{array}{l}\text { Bacterial } \\
\text { isolates }\end{array}$ & $\begin{array}{c}\text { No. of } \\
\text { isolates } \\
\text { screened }\end{array}$ & $\begin{array}{c}\text { No. resistant to } \\
\text { meropenem (Zones } \\
\mathbf{2 1 1 m m})\end{array}$ & $\begin{array}{c}\text { No. resistant to } \\
\text { imipenem } \\
\text { (Zone<23mm) }\end{array}$ & $\begin{array}{c}\text { No resistant } \\
\text { to both }\end{array}$ \\
\hline E. coli & 140 & $66(47.1)$ & $43(30.7)$ & $38(27.1)$ \\
K. pneumoniae & 108 & $34(31.4)$ & $48(44.4)$ & $19(17.6)$ \\
Total & $\mathbf{2 4 8}$ & $\mathbf{1 0 0 ( 4 0 . 3 )}$ & $\mathbf{9 1 ( 3 6 . 6 )}$ & $\mathbf{5 7}(\mathbf{2 2 . 9 )}$ \\
\hline
\end{tabular}

Of the total 191 carbapenem-resistant isolates screened for carbapenemase production, 41 $(37.6 \%)$ and $52(63.4 \%)$ of $E$. coli and $K$. pneumoniae respectively, produces carbapenemase. Furthermore, out of the total 93 isolates that were carbapenemase screened positive, $38.7 \%$ were MBL producers. Some of the isolates had the intrinsic capacity to produce two or three of the enzymes. Combined production of ESBL and carbapenemase was detected in $19 E$. coli and $16 K$. Pneumoniae and a combination of ESBL, carbapenemase and MBL were detected in $13 E$. coli and $11 K$. pneumoniae (Table 2 ).

The majority $(109,75.7 \%)$ of the 144 ESBL producers and $39(53.4 \%)$ of the 73 carbapenemase producers were from surgical wards, followed by from inpatients from other wards $(20.1 \%$ E. coli and $46.5 \% \mathrm{~K}$. pneumoniae) then outpatients with only $2.8 \%$ ESBL but no carbapenemase. About $63.5 \%(61 / 96)$ of the isolates from urine samples

DOI: http://dx.doi.org/10.4314/ejhs.v27i1.2 
Table 2: Prevalence of ESBL and carbapenemase-MBL mediated carbapenem resistance

\begin{tabular}{lcccccccc}
\hline $\begin{array}{c}\text { Bacterial } \\
\text { Species }\end{array}$ & \multicolumn{2}{c}{ Screening for ESBL } & \multicolumn{2}{c}{$\begin{array}{c}\text { Screening for } \\
\text { carbapenemase } \\
\text { production }\end{array}$} & $\begin{array}{c}\text { Screening of } \\
\text { carbapenemase producers } \\
\text { (CP) for MBL }\end{array}$ & $\begin{array}{c}\text { Combination of CP, } \\
\text { MBL and ESBL }\end{array}$ \\
\cline { 2 - 8 } & $\begin{array}{c}\text { No. } \\
\text { screene } \\
\text { d }\end{array}$ & $\begin{array}{c}\text { No positive } \\
\text { for ESBL }\end{array}$ & $\begin{array}{c}\text { No } \\
\text { screene } \\
\text { d }\end{array}$ & $\begin{array}{c}\text { No positive for } \\
\text { carbapenemas } \\
\text { e (\%) }\end{array}$ & $\begin{array}{c}\text { No of CP } \\
\text { screened }\end{array}$ & $\begin{array}{c}\text { No positive } \\
\text { for MBL }(\boldsymbol{\%})\end{array}$ & $\begin{array}{c}\text { CP+ESB } \\
\text { L }\end{array}$ & $\begin{array}{c}\text { CP+MBL+ } \\
\text { ESBL }\end{array}$ \\
\hline E. coli & 140 & $76(54.2)$ & 109 & $41(37.6)$ & 41 & $19(46.3)$ & $19(13.6)$ & $13(9.2)$ \\
K. pneumoniae & 108 & $68(62.9)$ & 82 & $52(63.4)$ & 32 & $17(53.1)$ & $16(14.8)$ & $11(10.1)$ \\
Total & $\mathbf{2 4 8}$ & $\mathbf{1 4 4}(\mathbf{5 8 . 0})$ & $\mathbf{1 9 1}$ & $\mathbf{9 3}(\mathbf{4 8 . 7})$ & $\mathbf{9 3}$ & $\mathbf{3 6}(\mathbf{3 8 . 7})$ & $\mathbf{3 5}(\mathbf{1 4 . 1 )}$ & $\mathbf{2 4}(\mathbf{9 . 6})$ \\
\hline
\end{tabular}

Note: figures in parenthesis are percentages

were ESBL positive, $57.3 \%$ (47/82) of isolates from cathertipstips samples were also ESBL positive while $43.1 \%(22 / 51)$ wound swabs and $75.6 \%(14 / 19)$ of the abscesses samples were ESBL positive (Table 3). Carbapenemase producers from urine, cathetertips and wound swabs followed a similar pattern with ESBL with being the highest in urine $(33.3 \%)$, followed by catheter tips $(15.8 \%)$ and wound swabs $(9.8 \%)$.

Table 3: Distribution carbapenemase, MBL and ESBLs producers among clinical samples

\begin{tabular}{lcccccc}
\hline Clinical samples & No. screened & CP & MBL & ESBL & CP+ESBL & CP+MBL+ESBL \\
\hline Urine & $96(38.7)$ & $32(33.3)$ & $18(18.7)$ & $61(63.5)$ & $15(15.6)$ & $8(8.3)$ \\
Catheter tips & $82(33.0)$ & $26(31.7)$ & $13(15.8)$ & $47(57.3)$ & $14(17.0)$ & $13(15.8)$ \\
Wound discharge & $51(20.5)$ & $12(23.5)$ & $5(9.8)$ & $22(43.1)$ & $6(11.7)$ & $3(5.8)$ \\
Abscesses fluid & $19(7.6)$ & $3(15.7)$ & $0(0.0)$ & $14(73.6)$ & $0(0.0)$ & $0(0.0)$ \\
Total & $\mathbf{2 4 8 ( 1 0 0 )}$ & $\mathbf{7 3 ( 2 9 . 4 )}$ & $\mathbf{3 6}(\mathbf{1 4 . 5})$ & $\mathbf{1 4 4 ( 4 1 . 1 )}$ & $\mathbf{3 5 ( 1 4 . 1 )}$ & $\mathbf{2 4 ( 9 . 6 )}$ \\
\hline
\end{tabular}

Note: figures in parenthesis are percentages. $\mathrm{CP}=$ carbapenemase

Antibacterial susceptibility of all the isolates resistant to IPM and MEM, including those that produced ESBL and or carbapenemase to selected members of the class: polymyxin, glycylcycline, and flouroquinolones were determined. Susceptibility of E. coli, and K. pneumoniae to colistin and tigecycline, irrespective of type of $\beta$ - lactamase they produced, was higher compared with their susceptibility to flouroquinolones. However, K. pneumoniae was less susceptible to colistin $(62.5 \%)$ and tigecycline $(67.5 \%)$ compared with E. coli $(66.7 \%$ and $72.6 \%)$, but with a little higher susceptibility to flouoroquinoles than E. coli (Table 4).

Table 4: Susceptibility of carbapenemase (MBL inclusive) and ESBL producing isolates to selected polymyxin, glycylcycline, and flouroquinolones

\begin{tabular}{lccccc}
\hline Bacterial Species & \multicolumn{4}{c}{ Antimicrobial Susceptibility } \\
\cline { 2 - 6 } & Colistin & Tigecycline & Ciprofloxacin & Levofloxacin & Ofloxacin \\
\cline { 2 - 6 } & No.susceptible & No. susceptible & No. susceptible & No. susceptible & No. susceptible \\
& $(\boldsymbol{\%})$ & $(\boldsymbol{\%})$ & $(\boldsymbol{\%})$ & $(\boldsymbol{\%})$ & $(\boldsymbol{\%})$ \\
\hline E. coli (117) & $78(66.7)$ & $85(72.6)$ & $57(48.7)$ & $63(53.8)$ & $43(36.7)$ \\
K.pneumoniae(120) & $75(62.5)$ & $81(67.5)$ & $77(64.1)$ & $53(44.1)$ & $60(50.0)$ \\
Total (237) & $153(64.6)$ & $166(70.0)$ & $134(56.5)$ & $116(48.9)$ & $103(43.4)$ \\
\hline
\end{tabular}

DOI: http://dx.doi.org/10.4314/ejhs.v27i1.2 


\section{DISCUSSION}

The occurrence and continual spread of ESBL and AmpC mediated beta lactam resistance in many hospitals in Nigeria is known only to few. The rise in treatment failures in low income countries with over stretched healthcare facilities, typical of those in the study area, has continued to make life difficult for patients, their relatives and the practicing HCWs. In addition to inappropriate and personal prescriptions of antibiotics, lack of proper and sustainable infection prevention and control (IPC) measures in place has made the situation worse. In the study, it was observed that nearly all of the isolates were either totally resistant or slightly susceptible to commonly prescribed antibiotics in the two hospitals. The first and second line treatment drugs such as amoxicillin, augmentin, ceftazidime, cefpodoxime and ceftriaxone are now fast approaching clinically irrelevance drugs (data not shown).

Carbapenems are considered to be one of the antibiotics of last resort for treatment of infections caused by multi drug resistant bacteria such as $E$. coli and $K$. pneumoniae (14). Alarms have been raised over and over on the dangers of spreading of carbapenem resistant bacteria in hospitals and communities, where they can be acquired if IPC programs are not in place. A survey of the two hospitals showed that usage of carbapenems is limited, probably due to their non-availability or there high cost. In hospital A, carbapenems are reserved only for very stubborn infections. Similarly, specific screening for highly resistant pathogens such as ESBL, AmpC producers are not conducted currently in the laboratories of the two hospitals, despite the several local reports on their existence and continual rise in the hospitals (1517). While the traditional antibiotic susceptibility testing technique in use has failed to detect them, the spread continues undetected and false diagnosis is being issued. In an ideal situation, isolates screened positive for ESBL or carbapenemase phenotypically need to be confirmed by a polymerase chain reaction-based molecular test, but this technique is not really available in routine laboratory investigations in the study area. Therefore, the study restricted the screening to phenotypic confirmation tests.
The prevalence of ESBL and carbapenemase among the isolates was 38.4 and $39.2 \%$ respectively. This is very high when compared with previous figures of $14.4 \%$ and $11.9 \%$ respectively reported in 2011 in same hospitals combined. Patients colonised with $\beta$ lactamresistant ESBL, AmpC or carbapenemase KPC producers could be an important source for further propagation of these pathogens among patients.

The carbapenem susceptibility of various pathogens obtained from various clinical samples of patients, irrespective of their ailment, decreased compared with previous reports of carbapenem resistance in none ICUs isolates in 2011 in which IPM susceptibility in Kano was $92,6 \%$ (18). The decrease of carbapenem susceptibility in few years in our study is worrisome given the very disturbing fatality ratio of 33.3:9 for patients infected with imipenem-resistant KPC producing Enterobacte $r \mathrm{spp}$ and those infected with imipenem-susceptible strains as reported by Marchaim et al. (19). In the case of the two hospitals and many others in the country and the entire region, mortality data regarding use of any antibiotic is lacking or at least inadequate.

E. coli was more resistant to MEM than to IMP while, in $K$. pneumoniae, the reverse was the case. Poor hygienic practices by HCWs are some of the driving forces of carbapenem resistance in our hospitals despite their low or no usage. The resistant genes in these pathogens can be easily transmitted to other patients in the same or other wards if proper care is not taken. The concurrent production of the duo makes the whole scenario more disturbing. However, the remaining 98 isolates of the 191 carbapenem resistant isolates that are not carbapenemase producers were treated as carbapenemase non-producers but were resistant to carbapenems and third generation cephalosporins probably due to production of ESBLs in association with impaired permeability via loss of porins. About $48 \%$ of the carbapenemase producers, produced MBLs, the most prevalent type reported in different countries as a source of several nosocomial outbreaks (8).

B-lactamase mediated enterobacterial infections in this study were found in almost all the samples from the patients, which suggest that the $\beta$-lactamase producers seem not to be specific

DOI: http://dx.doi.org/10.4314/ejhs.v27i1.2 
to sites, tissue or organs. This coincides with the view of Nordmann et al. 2009 (8). However, high prevalence was observed in patients with urinary tract related problems which may involve use of invasive devices such as catheter. In addition, since previous treatment with carbapenems was ruled out, other risk factors that might be associated with the acquisition of these bacteria in the hospitals are prolonged hospitalisations, ICU stay, and immune-suppression as gathered from their history. On the contrary, other studies attributed carbapenem-resistant $K$ pneumoniae infections in patients to previous therapy with carbapenem (20) or increased use of carbapenems in a hospital (21). All attempts to link the hospitalised patients from hospital A to B or vice versa were unsuccessful, making the common source of infection still unclear to us. The possibility of community acquisition is also not ruled out due to the lifestyle of most of the patients. Factors like consumption of foodstuffs or animals treated with antibiotics, or frequent patient contact with healthcare facilities are all possibilities. Only five of the patients had history of previous travel to India, one of the endemic areas in the world and place of birth of NDM-1. Three (3) of the patients only studied in India and had never have access to any hospitals except the initial medical examination which was carried out in school clinics. Only one (1) was treated earlier in India for an unconfirmed illness. In all the patients, the overall history of recent hospitalization was just $5.6 \%(14 / 248)$. Rural dwellers constituted $20.9 \%(52 / 248)$ of the total patients, where they might have been exposed to any of the resistant strains due to multiple antibiotic treatments in local nursing homes due to the unrestricted over-the-counter availability of drugs or self-medication.

Antibiotic treatment options for carbapenemresistant bacteria are limited and are highly costly where available. However, combination therapy with active drugs such as colistin, tigecycline, and flouroquinolones has been recommended (22). Susceptibility to colistin and tigecycline was still promising irrespective of the type of beta lactamase they produced. While the expanded spectrum of tigecycline has been reported to produced $100 \%$ susceptibility invitro against many
Enterobacteriaceae producing ESBLs and carbapenemase, treatment failures invitro have also been recorded. Similarly, colistin susceptibility and resistance in carbapenemase, ESBL and MBLs producing Enterobacteriaceae has been observed and reported previously. Despite their high activity in treating most of the uro-pathogens invitro in this study, tigecycline usage is limited in treating urinary tract infections in vivo due to its low urine concentration while colistin-polymyxins member have high neurotoxicity and nephrotoxicity (8). Their successful use in vivo was not documented in this study.

There is a need for urgent intervention in our hospitals as the data generated on increasing antibiotic resistance from 2007 to-date suggest. It is envisaged for the next decade that the emergence of NDM, MBL, XDR and KPC in our health care facilities will significantly escalate healthcare costs associated with laboratory detection and salvage combination therapy for infections due to these pathogens.

It is concluded that carbapenemase-ESBL mediated carbapenem resistant pathogens have occurred among the pathogens commonly isolated in the two tertiary hospitals in Nigeria. Coproduction of two or more $\beta$ lactamase occurred among some of the isolates, raising fears of resistance to a multitude of antibiotics in the treatment of clinical infections. There is a sharp decrease in carbapenem susceptibility among the pathogens despite low carbapenem usage in the hospitals, which can make treatment of infections caused by these pathogens difficult. Colistin and tigecycline are still promising while resistance to flouroquinolones, which are mostly used in the hospitals, is increasing.

\section{REFERENCES}

1. Neuchauser, MM, Weinstan RA, Rydman IR, Danziger LH, Karam G, Quinn JP. Antibiotic resistance among Gram negative Bacilli in US intensive care units: implications for flouroquinolonesuse JAMA 2003;289: 885-8.

2. Paterson DL, WC KO, Goossens H. Antibiotic therapy for Klebsiella pneumoniae bacteremia. Implication of production of extended spectrum Blactamases. J Clin Microbiol 2004; 39(5): 50-57.

DOI: http://dx.doi.org/10.4314/ejhs.v27i1.2 
3. Rhomberg PR, Jones RN, Jones RN. Contemporary activity of meropenem and comparator broad spectrum agents: MYSTIC program report from the United States component. DiagnMicriobiol infect 2007; 57: 207-15.

4. Winokur PC, Cantoon R, Caseilas JM, Legakis N. Variations in the prevalence of strains expressing and extended spectrum beta-lactamase phenotype and characterization of isolates from Europe, the America's and the western pacific regions. Clin infect Dis 2001;32 suppl 2: 94-103.

5. Lee HS, Jang SJI, Park AJ, Lem M, Song WK. Antimicrobial resistance surveillance of bacterial in 1999 in Korea with a special reference to resistance of Enterococci to vancomycin and Gram Negative Bacilli to third generation cephalosporins, imipenem, and flouroquinolones. J. Korean Med Sci2001;16: 262-70.

6. Dubois V, Arpin C, Melon M, Melon B, Andre C, Frigo C. Nosocomial outbreak due to multidrug resistant strain of Pseudomonas aeruginosaP12: efficacy of cefepime-amikacin therapy and analysis of beta-laclam resistance. J Clin Microbol2001; 39: 2072-8.

7. Tsakris A, Poulou A, Pournaras S, et al. A simple phenotypic method for the differentiation of metallo- $\beta$-lactamases and class A KPC carbapenemase in Enterobacteriaceae clinical isolates. J Antimicrob Chemother2010; dkq210.

8. Nordmann P, Cuzon G, Naas T. The real threat of Klebsiella pneumoniae carbapenemase-producing bacteria. Lancet Infect Dis2009;9(4): 228-236.

9. Fernando P, Tania M, Leonor G, Melina R, Alejandra C. Sensitive screening tests for suspected class A carbapenemase production in species of Enterobacteriaceae. J. ClinMicrobiol2009; 47(6): 1631-39.

10. Jones KE, Patel NG, Levy MA, et al. Global trends in emerging infectious diseases. Nature 2008;451(7181): 990-93.

11. Clinical Laboratory Standards institute. Current Clinical and Laboratory Standards Institute (CLSI). Criteria for interpretation of susceptibility testing of carbapenems in Enterobacteriaceae. CLSI document M100, S22 2012.

12. CDC. Guidance for control of infections with carbapenem-resistant or carbapenemase producing Enterobacteriaceae in acute care facilities. MMWR Weekly 2009; 58(10): 256-260.
13. Walsh TR, Toleman MA, Poirel L, Nordmann P. Metallo-beta-lactamases: the quiet before the storm? ClinMicrobiol Rev 2005;18: 306-325

14. Bush K, Jacoby CA, Medeiros AA. A functional classification scheme for beta-lactamases and its correlation with molecular structure.Antimicrob Agent Chemother1995; 39: 1211.

15. Yusuf I, Yusha'u M, Sharif AA, Getso MI, Yahaya H, Bala JA, Aliyu IA, Haruna M. Detection of Metallobetalactamases among Gram negative bacterial isolates from Murtala Muhammad Specialist Hospital, Kano and Al-madina Hospital Kaduna, Nigeria. Bayero J Pure Appl Sci 2012; 5(2): 84-88.

16. Yusuf I, Arzai AH, Haruna M, Sharif AA, Getso, MI. Detection of multi drug resistant bacteria in major hospitals in Kano, North-West, Nigeria. Braz J Microbiol 2014; 45(3), 791-798.

17. Yusha'u M, Olonitola SO, Aliyu BS. Prevalence of Extended-Spectrum B- lactamases (ESBLs) among members of the Enterobacteriaceae isolates obtained from Mohammed AbdullahiWase Specialist Hospital, Kano, Nigeria. Int J Pure App Sci 2007; 1(3): 42-48.

18. Yusuf I, Haruna M, Yahaya H. Prevalence and antibiotic susceptibility of AmpC and ESBL producing clinical isolates at a tertiary health care center in Kano, Northwest Nigeria. Afr J Clin Exper Microbiol 2013; 14(2): 109-109

19. Marchaim D, Navon-Venzia S, Schwaber MJ, et al. Isolation of imipenem-resistant Enterobacterspecies; emergence of KPCcarbapenemase, molecular characterization, epidemiology and outcomes. Antimicrob Agents Chemother2008; 52:1413-18.

20. Bratu S, Landman D, Haag R, et al. "Rapid spread of carbapenem-resistant Klebsiella pneumoniae in New York City:a new threat to our antibiotic armamentarium."Arch Intern Med 2005; 165:1430-35.

21. Coetzee L, Brink A. The emergence of carbapenem resistance in Enterobacteriaceae in South Africa.Southern Afr JEpid Infect 2011; 26: 239240.

22. Kanj SS, Kanafani ZA. Current concepts in antimicrobial therapy against resistant Gram negative organisms: Extended-spectrum $\beta$ lactamase producing Enterobacteriaceae, carbapenem-resistant Enterobacteriaceae, and multi-drug resistant Pseudomonas aeruginosa. Mayo ClinProc2011;86(3):250-259. 